$$
\begin{aligned}
& U==\left[2\left(x^{2}+y^{2}\right)\right]^{-1 / 2}\left(\begin{array}{ccc}
x-y & x+y & 0 \\
x+y & y-x & 0 \\
0 & 0 & {\left[2\left(x^{2}+y^{2}\right)\right]^{1 / 2}}
\end{array}\right) \\
& c^{*}=a^{*} U=-(2)^{-1 / 2}\left(x^{2}-y^{2}\right)^{-3 / 2} \dot{R}(-z(x+y), z(y-x), \\
&\left.x\left[2\left(x^{2}+y^{2}\right)\right]^{1 / 2}\right) .
\end{aligned}
$$

Therefore,

$$
\begin{aligned}
&\left(x^{2}+y^{2}\right) \psi=y_{2}{ }^{2}-y_{1}{ }^{2}+ \dot{R}(2)^{1 / 2}\left(x^{2}+y^{2}\right)^{-1 / 2} \\
& \cdot\left(-z(x+y) y_{1}+z(y-x) y_{2}+x\left[2\left(x^{2}+y^{2}\right)\right]^{1 / 2} y_{3}\right) .
\end{aligned}
$$

After neglecting the fixed factor $x^{2}+y^{2}$,

$$
\frac{2 g(\lambda)}{\dot{R}^{2}}=\frac{z^{2}(x+y)^{2}}{\left(x^{2}+y^{2}\right)(\lambda+1)^{2}}+\frac{z^{2}(y-x)^{2}}{\left(x^{2}+y^{2}\right)(\lambda-1)^{2}}+\frac{2 x^{2}}{\lambda^{2}} .
$$

In the general case, when none of the numerators are zero, the problem is solved by finding the largest real root of $(3.8)$ with $g(\lambda)=1$. Classical root calculation procedures, such as Newton's method, should encounter no difficulty. If one or more of the numerators are zero, the computation is simpler. For example, if $z=0$, $g(\lambda)=\dot{R}^{2} x^{2} / \lambda^{2}$ and Case I applies if $\lambda=|\dot{R} x| \geqq 1$. Then $y_{1}=y_{2}=0, y_{3}= \pm 1$; if $|\dot{R} x|<1$, then Case II applies and $y_{1}=0, y_{2}=\left(1-\dot{R}^{2} x^{2}\right)^{1 / 2}, y_{3}=\dot{R} x$. The geometric interpretation of this is that the Coriolis term predominates for large $x$ values.

1. George E. Forsythe \& Gene H. Golub, Maximizing a second-degree polynomial on the unit sphere, Tech. Rep. CS16, Stanford University Computer Science Department, Stanford, Calif., 1965.

\title{
Questions Concerning Khintchine's Constant and the Efficient Computation of Regular Continued Fractions
}

\author{
By John W. Wrench, Jr. and Daniel Shanks
}

Let $x$ be a real number whose regular continued fraction is given by

$$
x=a_{0}+\frac{1}{a_{1}}+\frac{1}{a_{2}}+\frac{1}{a_{3}}+\ldots,
$$

with $a_{0}$ an integer, and $a_{1}, a_{2}, a_{3}, \cdots$ positive integers. Let

$$
G_{n}(x)=\left(a_{1} \cdot a_{2} \cdot a_{3} \cdot \cdots \cdot a_{n}\right)^{1 / n} .
$$

Then Khintchine's famous theorem states that, for almost all $x$,

$$
\operatorname{Lim}_{n \rightarrow \infty} G_{n}(x)=K,
$$

Received January 24, 1966. 
where $K$ is an absolute constant given by

$$
K=2.685452001 \cdots .
$$

"Almost all" means, of course, except for a set of measure zero. Contained in this set of measure zero are, however, all rational numbers, all quadratic surds: $\left(n_{1}+\left(n_{2}\right)^{1 / 2}\right) / n_{3}$, the number $e$, since

$$
e=2+\frac{1}{1}+\frac{1}{2}+\frac{1}{1}+\frac{1}{1}+\frac{1}{4}+\frac{1}{1}+\frac{1}{1}+\frac{1}{6}+\frac{1}{1}+\frac{1}{1}+\frac{1}{8}+\ldots,
$$

and presumably much more. In a recent review [1] of two translations of Khintchine's book Continued Fractions, the second-named author had occasion to make the ironic inquiry as to whether Khintchine's constant $K$ is itself in the "almost all". The adjective "ironic" seems entirely appropriate here regardless of the (as yet unknown) answer to this question, for if $K$ is not in the "almost all", the situation is truly ironic, in fact, one even might think, unjust; whereas if $K$ is in the "almost all", one has the ironic situation wherein $K$ to a million decimals, say, would have a continued fraction from which one could recover $K$ to, say, three decimals.

Several years ago, this second author had computed the first 30 partial quotients, $a_{0}$ to $a_{30}$ :

$$
K \approx 2+\frac{1}{1}+\frac{1}{2}+\frac{1}{5}+\cdots+\frac{1}{1}
$$

using the value of $K$ given in [2]. He found that they seemed smaller, in the (geometric) mean, than is usually the case, and were such that

$$
G_{30}(K)=2.126 .
$$

Put another way, the rational approximations to $K$, with denominators that are not too large, are not quite as good as those for almost all numbers. It seemed not unreasonable, however, that this anomaly, based on the early approximations, could well disappear as later approximations were examined. But the problem seems to us quite infeasible theoretically, either now or in the near future.

The first-named author has now tested this supposition by computing the first 150 partial quotients, utilizing his [3] more accurate value of $K$. In Table 1, we list the partial quotients $a_{n}$ for $n=0(1) 150$, and in Table 2 the geometric means, $G_{n}(K)$, and the deviations, $G_{n}(K)-K$, for $n=10(10) 150$. It will be noted that the above-described anomaly is very persistent, and only disappears at the very end of our table. Of course, it is clear that nothing conclusive can be determined about the main question in this way, but at least it is fair to say that we no longer have any reason to believe that $K$ is not in the "almost all".

We now turn to the question of computing the $a_{i}$ in (1) efficiently, given some $x$ to many decimal places. The most efficient method known has already been published [4], and it may seem that nothing more need be said. Nonetheless, it is a fact that this method is not well-known, and that many investigators have used, and continue to use, methods that are more obvious, but which require far more arithmetic, cf. [5]. Since this journal has as one of its purposes the analysis of efficient computation, it seems desirable to examine here the questions of why these less efficient methods are used, and wherein lies their inefficiency. 
TABLE 1

Partial quotients in the regular continued fraction for Khintchine's constant

\begin{tabular}{r|r|r|r|r|r|r|r|r|r|r}
\hline$n$ & \multicolumn{1}{r|}{0} & 1 & 2 & 3 & 4 & 5 & 6 & 7 & 8 & 9 \\
\cline { 2 - 9 } 0 & 2 & 1 & 2 & 5 & 1 & 1 & 2 & 1 & 1 & 3 \\
1 & 10 & 2 & 1 & 3 & 2 & 24 & 1 & 3 & 2 & 3 \\
2 & 1 & 1 & 1 & 90 & 2 & 1 & 12 & 1 & 1 & 1 \\
3 & 1 & 5 & 2 & 6 & 1 & 6 & 3 & 1 & 1 & 2 \\
4 & 5 & 2 & 1 & 2 & 1 & 1 & 4 & 1 & 2 & 2 \\
5 & 3 & 2 & 1 & 1 & 4 & 1 & 1 & 2 & 5 & 2 \\
6 & 1 & 1 & 3 & 29 & 8 & 3 & 1 & 4 & 3 & 1 \\
7 & 10 & 50 & 1 & 2 & 2 & 7 & 6 & 2 & 2 & 16 \\
8 & 4 & 4 & 2 & 2 & 3 & 1 & 1 & 7 & 1 & 5 \\
9 & 1 & 2 & 1 & 5 & 3 & 1 & 1 & 1 & 2 & 2 \\
10 & 2 & 1 & 13 & 11 & 770 & 1 & 4 & 2 & 1 & 14 \\
11 & 1 & 14 & 2 & 1 & 6 & 1 & 1 & 1 & 9 & 2 \\
12 & 53 & 1 & 2 & 2 & 1 & 9 & 5 & 6 & 2 & 1 \\
13 & 2 & 1 & 5 & 4 & 1 & 234 & 7 & 1 & 1 & 4 \\
14 & 3 & 19 & 3 & 1 & 10 & 18 & 8 & 24 & 1 & 12 \\
15 & 1 & & & & & & & & & \\
\hline
\end{tabular}

TABLE 2

\begin{tabular}{r|c|c}
\hline$n$ & $G_{n}(K)$ & $G_{n}(K)-K$ \\
\cline { 2 - 3 } 10 & 1.89590 & -0.78955 \\
20 & 2.11174 & -0.57371 \\
30 & 2.12606 & -0.55939 \\
40 & 2.22084 & -0.46461 \\
50 & 2.10320 & -0.58225 \\
60 & 2.02209 & -0.66336 \\
70 & 2.18395 & -0.50150 \\
80 & 2.37681 & -0.30864 \\
90 & 2.34452 & -0.34093 \\
100 & 2.27432 & -0.41113 \\
110 & 2.44835 & -0.23710 \\
120 & 2.51097 & -0.17448 \\
130 & 2.49488 & -0.19057 \\
140 & 2.56242 & -0.12303 \\
150 & 2.69503 & +0.00958 \\
\hline
\end{tabular}

The obvious method of computing (1) is to set

$$
\alpha_{0}=x, \quad a_{n}=\left[\alpha_{n}\right], \quad \alpha_{n+1}=\frac{1}{\alpha_{n}-a_{n}},
$$

with $[y]$ the integer part of $y$. The main arithmetic is the multi-precision division in the third equation of (8). Since this division requires a number of operations proportional to the square of the number of decimal places, it transpires that the expansion of (1) up to $a_{n}$, in this way, requires arithmetic proportional to $n^{3}$, cf. [5, p. 278].

In contrast, consider $x$ to be bounded by two rational numbers: 


$$
\frac{\beta_{0}}{\beta_{1}}<x<\frac{\gamma_{0}}{\gamma_{1}}
$$

for example

$$
\frac{2685452001}{1000000000}<K<\frac{2685452002}{1000000000} .
$$

Then define

$$
\begin{aligned}
c_{n} & =\left[\frac{\gamma_{n}}{\gamma_{n+1}}\right], & \gamma_{n+2} & =\gamma_{n}-c_{n} \gamma_{n+1}, \\
b_{n} & =\left[\frac{\beta_{n}}{\beta_{n+1}}\right], & & \beta_{n+2}=\beta_{n}-b_{n} \beta_{n+1},
\end{aligned}
$$

and, it is easily seen, as long as $b_{n}=c_{n}$, they also equal the $a_{n}$ of (8). This is, in fact, nothing but Euclid's Algorithm. Now, since $c_{n}\left(b_{n}\right)$ are almost always singleprecision numbers, the multiplications and multi-precision subtractions in (10) require arithmetic proportional to the number of decimal places, and the previous $n^{3}$ behavior is replaced here by only $n^{2}$.

We now think it pertinent to ask two questions, one psychological, and one logical:

(a) Why is it that computers have repeatedly, as in [5], used (8) rather than the more efficient (10), even if they had much computing experience, and probably also knew of Euclid's Algorithm?

(b) Wherein lies the loss of efficiency in (8)? On the face of it, it appears to be perfectly straightforward, and to involve no unnecessary operations.

We believe the answer to (a) is this. The computer, when dealing with a highprecision irrational $x$ finds it more congenial to imagine $x$ as an "infinite" decimal number $\alpha_{0}$, as in (8), rather than as (the logically more exact) sequence of nested rational intervals, as exemplified in (9). It seems simpler to him, since only one $\alpha$ is needed, instead of two $\beta$ 's, $\lambda$ and two $\gamma$ 's. Further, his experience as a computer does not save him from the trap. On the contrary. He notes that the main problem in (8) is to devise an efficient technique of carrying out the multi-precision division, and, since he is probably proud of his computing skill, he now exerts himself in this direction, and then feels some satisfaction in whatever he accomplishes there.

The answer to (b), which has profited at least some by being discussed with Professor Hans Zassenhaus, is this. In (8), we not only obtain the wanted quantities, $a_{i}$, but we also obtain the unneeded decimal expansions of the $\alpha_{i}$. For example, if $\alpha_{0}=K$, we have

$$
\begin{aligned}
& \alpha_{0}=2.685452001 \cdots, \\
& \alpha_{1}=1.458891357 \cdots, \\
& \alpha_{2}=2.179165034 \cdots, \text { etc. }
\end{aligned}
$$

This is where the extra effort has gone to. If we wished to obtain these decimal expansions from (10) we would need to carry out the multi-precision divisions of $\gamma_{n} / \gamma_{n+1}=\alpha_{n}$.

Abstractly speaking, inefficient computations, (or the analogously similar, un- 
necessarily long proofs of theorems), are usually due to one of two phenomena: First, there is a true redundancy, whereby two phases of the computation (proof) merely cancel each other out. See [6] for the analysis of such a theorem proof. Secondly, the computation (proof) does more than is really needed, or wanted. See [7] for such a theorem proof. In our present case the inefficiency is clearly of the second type.

Finally, we note that D. H. Lehmer, in [4], showed how most of the subtractions in (10) could also be eliminated. It is not necessary to compute every $\gamma_{n} / \gamma_{n+1}$, but merely those at periodic intervals. It was his method that was used here in computing Table 1. Any interested reader will now have no difficulty in determining precisely how Lehmer manages to obtain this still greater efficiency.

David Taylor Model Basin

Washington, D. C. 20007

1. Daniel Shanks, (Reviewer), RMT 11, 12, (Review of two translations of Khintchine's Continued Fractions), Math. Comp., v. 20, 1966, pp. 171-173.

2. Daniel Shanks \& J. W. Wrench, JR., "Khintchine's constant," Amer. Math. Monthly, v. 66,1959 , pp. 276-279. MR $21 \# 1950$.

3. J. W. WRENCH, JR., "Further evaluation of Khintchine's constant," Math. Comp., v. 14, 1960 , pp. $370-371$.

4. D. H. Lehmer, "Euclid's Algorithm for large numbers," Amer. Math. Monthly, v. 45, 1938, pp. 227-233.

5. D. E. KNUth, "Euler's constant to 1271 places," Math. Comp., v. 16, 1962, pp. 275-281. MR $26 \# 5763$.

6. DANiel Shanks, "A study of postulates: The 'thermodynamic' derivation of the adiabatic gas law," Amer. J. Phys., v. 24, 1956, pp. 352-354.

7. Daniel Shanks, "Is the quadratic reciprocity law a deep theorem?" Solved and Unsolved Problems in Number Theory, Vol. 1, Spartan, Washington, 1962, p. 65, see (a). MR 28 $\# 3952$.

\section{Distributions of Mersenne Divisors}

\section{By Sidney Kravitz}

By driving computers to the limit of their capability, 23 prime Mersenne Numbers have been discovered [1]. The list of known divisors on the other hand is a large one. As a result of both of these lists, conjectures have appeared regarding the expected number of primes and of divisors [1], [2], [3]. This note presents additional data relative to the observed frequency of divisors of Mersenne Numbers.

Each divisor, $q$, of the Mersenne Number $M_{p}=2^{p}-1, p$ a prime, is of the form $2 k p+1$ and of the form $8 L \pm 1$. (Therefore $k \neq 4 n+2$.) Thus if $k$ is known for a particular $p$, it identifies the divisor. The divisors of the Mersenne Numbers, $3 \leqq p$ $<100,000$ have been examined for $k \leqq 200$. The frequency $f$, with which the various values of $k$ occur is given in Table 1. This table shows that the frequency of $k$ tends to decrease as $k$ increases, but those $k$ with a large number of small divisors, e.g. 12, 24, and 60, occur with much greater frequency than their neighbors on the list.

Received November 29, 1965. 